\title{
Du management de projets au management stratégique par projet : Les projets au service de la stratégie
}

\author{
Selma Katlane Ben Mlouka ${ }^{a}$ et Hajer Khedher Ouinniche ${ }^{b}$ \\ Université de Tunis El Manar
}

\section{INTRODUCTION}

Le management par projet est une approche opérationnelle pour la mise en œuvre des choix stratégiques basée sur la reconfiguration de l'organisation en processus projets.

Le fonctionnement par projet constitue un moyen favorisant la concentration sur les exigences du client pour le satisfaire et lui concevoir un produit ou un service à la limite «sur mesure », rapidement, grâce à des équipes projets. L'insertion de ces projets, qualifiés parfois de corps étrangers dans l'organisation, est source de changements organisationnels importants au point de les insérer dans une discipline spécialisée, soit le management par projet.

S'inscrivant dans le cadre du management stratégique de l'entreprise, le management par projet est une approche opérationnelle pour la mise en œuvre des choix stratégiques basée sur la reconfiguration de l'organisation en processus projets.

Le projet est une organisation et un processus transversal temporaire défini pour atteindre un objectif sous les contraintes de temps, de budget et d'autres ressources. De plus en plus d'entreprises font appel de manière plus ou moins intensive au mode d'organisation par les projets pour maintenir et/ou améliorer leurs positions compétitives. Plusieurs raisons permettent d'intégrer le projet dans le mode d'organisation des entreprises. La complexité de l'environnement, la réduction des cycles de vie des produits et la révolution des TIC ont favorisé la course des entreprises pour conserver leurs parts de marchés en œuvrant continuellement à relever plusieurs défis : une bonne qualité et une différenciation des produits, le respect des délais et la minimisation des coûts. À ce niveau, c'est le problème $d u$ «comment» que se posent les entreprises. L'organisation transversale, voire la gestion par les processus ou encore dans sa forme extrême le fonctionnement par projet, a le mérite de faciliter non seulement la réactivité au nouveau contexte, mais aussi de soutenir la créativité espérée par les organisations d'aujourd'hui ${ }^{1}$.

L'entreprise orientée projet est une entreprise caractérisée par une grande ouverture sur l'environnement interne et externe cherchant à s'approcher plus du client pour être la première à le servir et à le satisfaire. À cette fin, on introduit des changements organisationnels et structurels (une hiérarchie plate où les responsabilités sont réparties horizontalement autour du management par projet) ainsi que des changements culturels et comportementaux favorisant un état d'esprit collectif et un travail en équipes basé sur un système de communication formel et informel transversal dans l'organisation.

L'entreprise orientée projet est une entreprise caractérisée par une grande ouverture sur l'environnement interne et externe cherchant à s'approcher plus du client pour être la première à le servir et à le satisfaire.

Dans les entreprises orientées projet, la priorité est normalement donnée aux projets sur les structures métiers dans l'affectation des ressources. La structure projet, à sa tête un chef de projet, est autonome par rapport au reste de l'entreprise et les rôles sont répartis entre le «front office» et le «back office». Le front office étant composé par les acteurs du projet à travers lesquels la force motrice vient du marché, à la différence des démarches de type «top/down » où la force motrice est la direction générale. Quant au «back office», il constitue le 
réservoir de ressources et de compétences au niveau des métiers où la direction générale joue un rôle important en développant et en libérant les énergies et les compétences. L'objectif est d'adapter le modèle de management et de renouveler les pratiques de gestion en vue d'atteindre l'efficacité recherchée par le «front office », lequel est en contact avec le client et les fournisseurs. La répartition des rôles entre ces deux missions peut faire émerger des difficultés de nature à entraver le fonctionnement du management par projet.

Certains auteurs $^{3}$ différencient les entreprises orientées produits des entreprises orientées projets selon trois aspects: les processus de gestion, les processus techniques et la structure organisationnelle. Le tableau 1 présente une synthèse des différences entre ces deux catégories d'organisation.

Tableau 1 : Entreprise orientée produit versus entreprise orientée projet

\begin{tabular}{|c|c|c|}
\hline & Entreprise orientée produit & Entreprise orientée projet \\
\hline Processus de travail & Produire pour stoker & Produire à la commande \\
\hline Produit & Produit standard & Produit conforme aux spécificités \\
\hline Demande & Peut être prévue & Difficultés de prévision \\
\hline Prix & Prédéterminé & Déterminé à la commande \\
\hline Date de livraison & $\begin{array}{l}\begin{array}{l}\text { N'est pas stricte, fixée } \\
\text { disponibilité du stock }\end{array} \\
\text { dispon }\end{array}$ & Stricte, déterminée à la commande \\
\hline Equipement & Spécialisé & Universel \\
\hline Personnel & Savoir-faire spécialisé & Savoir-faire varié \\
\hline Processus technique & Production continue & Production discrète \\
\hline Spécialité & Pipe-line & Assemblage \\
\hline Production & De masse & A l'unité \\
\hline Degré d'automatisme & Elevé & Faible \\
\hline Répétitivité & Elevée & faible \\
\hline $\begin{array}{c}\text { Structure } \\
\text { organisationnelle }\end{array}$ & $\begin{array}{c}\text { Structure fonctionnelle ou } \\
\text { multidivisionnaire }\end{array}$ & Structure matricielle \\
\hline Principe & Spécialisation fonctionnelle & Coordination des équipes projet \\
\hline Avantage & Autorité & Flexibilité \\
\hline Désavantage & Rigidité & Double commandement \\
\hline Stratégie & $\begin{array}{l}\text { Stratégie fonctionnelle ou stratégie } \\
\text { d'activité }\end{array}$ & $\begin{array}{l}\text { Stratégie de portefeuille et stratégie } \\
\text { du projet }\end{array}$ \\
\hline
\end{tabular}

\section{DE LA GESTION DE PROJETS AU MANAGEMENT PAR PROJET}

\subsection{Le concept de gestion de projets}

Un projet est un processus ou un ensemble d'étapes qu'il s'agit de diriger, planifier, coordonner et contrôler pour la réalisation d'une finalité. La gestion de projets consiste à prendre en charge le plan d'action et le plan d'animation, en admettant que le fondement du projet soit bien établi. Elle revient à mettre l'accent sur les aspects humains et organisationnels : il s'agit d'animer un groupe, de planifier, de mettre en place un suivi, de communiquer, de prévoir un bilan dans une optique constructive. Il ressort ainsi que le projet comporte deux aspects : un aspect organisationnel et un aspect humain. Concernant l'aspect organisationnel du projet, il est utile de souligner que la gestion de projets est vue par beaucoup de professionnels et d'experts comme une méthodologie comportant trois étapes : la définition et la planification du projet (fixation des objectifs du projet, détermination du produit final avec le client et évaluation des besoins en ressources humaines, matérielles et financières), la réalisation ou la mise en place du 
projet et enfin son suivi et son contrôle ${ }^{5}$. Ainsi, l'aspect organisationnel du projet comporte la conduite des étapes de gestion de projets.

L'aspect humain, qui est loin d'être considéré comme une méthodologie, est relatif à la gestion des hommes, à la répartition et à la délégation du travail aux membres de l'équipe de projets et à la reconnaissance et au développement des compétences et des aptitudes des salariés de manière à favoriser leur créativité dans le projet. Finalement, on peut avancer que l'aspect humain dans la gestion de projets consiste à assurer la cohésion entre les membres de l'équipe de projets. L'introduction de cette nouvelle manière de «comment travailler» peut entraîner un changement dans l'entreprise susceptible de causer un effet perturbateur chez le personnel qu'il y a lieu de maîtriser en mettant en place les meilleures conditions permettant aux projets d'être pilotés. Il s'agit, en outre, d'insuffler à l'ensemble de l'entreprise, une culture projet qui privilégie le travail en groupes temporaires, transversaux et inter-fonctionnels chaque fois que des projets sont identifiés au niveau de l'entreprise. C'est là le rôle du management par projet qu'il convient de préciser.

\section{L'aspect humain dans la gestion de projet consiste à assurer la cohésion entre les membres de l'équipe de projet.}

\section{Le concept de management par projet}

Comme une discipline managériale formelle, le management par projet est encore relativement récent. Il a été formellement développé dans le milieu du $20^{\mathrm{e}}$ siècle quand le $1^{\mathrm{er}}$ programme PERT a marqué le commencement d'une nouvelle discipline. Avec le temps, les outils, les techniques et les méthodes sont devenues standardisées dans les industries et dans les affaires, et de plus en plus d'organisations commencent à témoigner des bénéfices de l'organisation du travail autour des projets. La formation de l'institut du management par projet (PMI) en 1969 comme une première association professionnelle a été un important pas dans le développement de la discipline.

\section{Dans un contexte de management par projet, les projets peuvent être gérés indépendamment ou combinés les uns par rapport aux autres selon les ressources matérielles, financières et humaines allouées par l'entreprise.}

Le management par projet est défini comme des activités managériales utiles pour conduire à la réussite du projet. Dans ce mode de gestion, les projets sont considérés comme autant d'unités distinctes à intégrer ensuite au sein de l'entreprise. Dans un contexte de management par projet, les projets peuvent être gérés indépendamment ou combinés les uns par rapport aux autres selon les ressources matérielles, financières et humaines allouées par l'entreprise. On peut ainsi distinguer les projets de valorisation technologique et de développement des compétences, les projets d'amélioration et de repositionnement des produits et services existants, les projets de conception ou de développement de nouveaux produits ou de nouvelles activités, les projets d'organisation interne, etc ${ }^{7}$.

Partant de ces définitions, on peut déjà soulever la différence entre le management par projet et la gestion de projets. En effet, la gestion de projets est une discipline qui concerne un seul projet comportant des outils, techniques et approches organisationnelles pour gérer les relations et les comportements humains. Le management par projet concerne un portefeuille de projets distincts à intégrer avec succès au sein de l'entreprise dans les meilleures conditions : établissement des priorités entre les projets, ainsi que des structures d'organisation adéquates. Cette dernière fonction attribuée au management par projet fait appel au concept de « gestion intégrative » introduit dans certains travaux de formalisation du management stratégique et repris dans la théorie de "l'intégration différenciation $^{8} »$. En effet, il y a lieu d'abord de rappeler que les activités de l'entreprise peuvent être classées en projets qui exigent une gestion de projets et en opérations qui font appel à des techniques du management des opérations. Le sommet stratégique, lequel est responsable de la définition de la stratégie et des objectifs de 
l'entreprise, est appelé à assurer dans les meilleures conditions, une allocation optimale des ressources entre les processus opérations et les processus projets pour la mise en place de la stratégie. La gestion stratégique se caractérise dans les trois fonctions suivantes: la gestion opérationnelle chargée du rendement des opérations courantes, la gestion innovative ou entrepreneuriale et en particulier la gestion de projets et la gestion intégrative. La gestion intégrative est statique lorsqu'il s'agit de faire cohabiter les systèmes de management et les cultures associées au projet et aux opérations en assurant une allocation optimale des ressources aux opérations et aux projets, l'évaluation des opportunités de projet, mais aussi et surtout la répartition des compétences managériales de l'organisation aux fins des projets et des opérations $^{9}$. La gestion intégrative est dyna- mique lorsqu'il faut passer un projet au stade d'opération stabilisée et rentable. En définitive, la gestion intégrative peut constituer le cadre théorique à travers lequel le management par projet est mis en place tel que présenté à la figure 1 .

Le sommet stratégique, lequel est responsable de la définition de la stratégie et des objectifs de l'entreprise, est appelé à assurer dans les meilleures conditions, une allocation optimale des ressources entre les processus opérations et les processus projets pour la mise en place de la stratégie.

Figure 1 : Gestion intégrative, gestion des projets et gestion des opérations

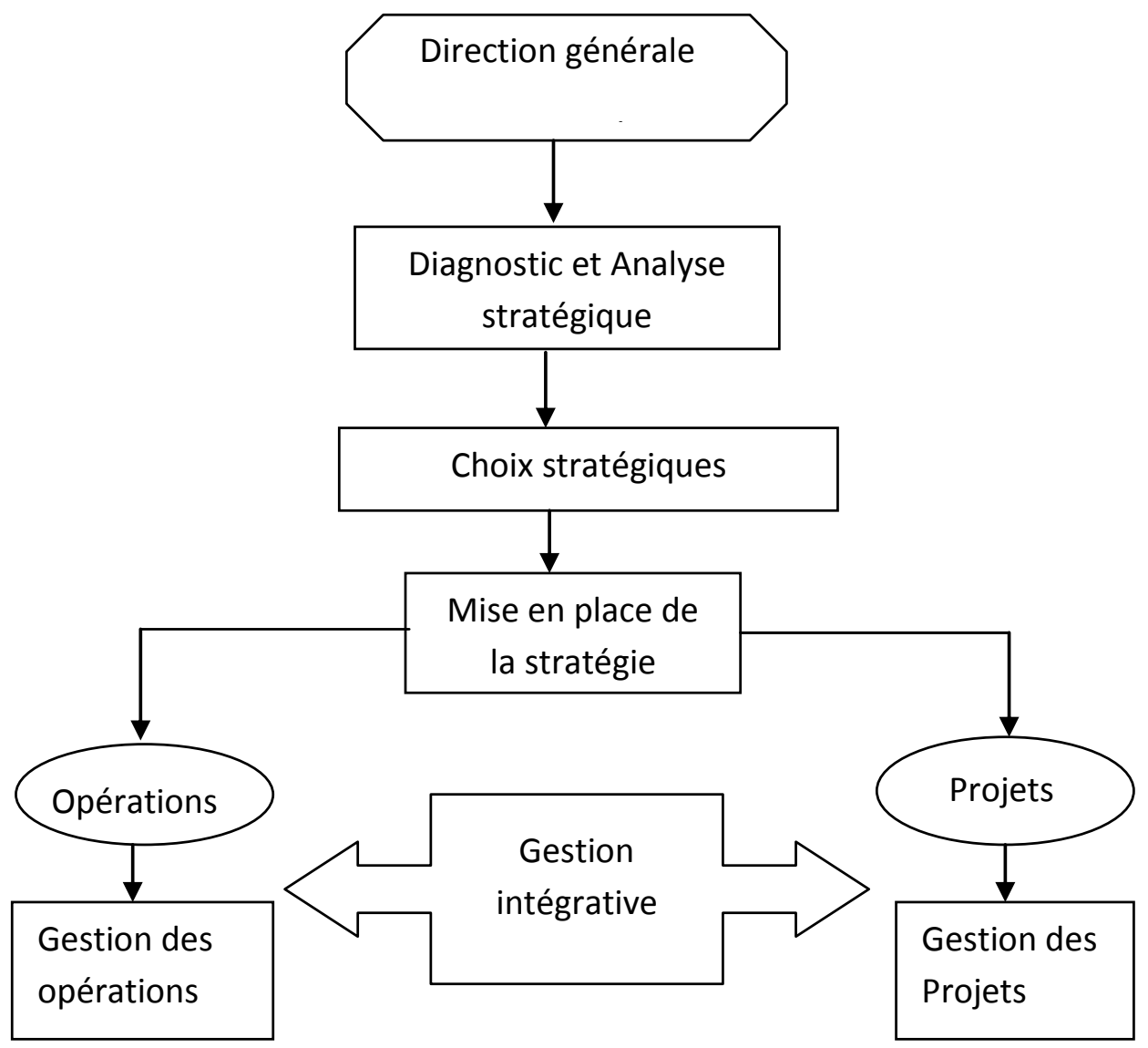


Aujourd'hui, avec les mutations de

l'environnement, la stratégie peut être mise en œuvre grâce à un portefeuille de projets sachant introduire et réaliser le changement espéré tant dans l'environnement externe que dans l'environnement interne.

Comme l'on noté Tarondeau et Wright, «la coopération transversale, inter-métiers ou interservices, agencée par processus ou par projet, est la traduction opérationnelle ou structurelle de stratégies visant à obtenir des avantages concurrentiels en termes de qualité et d'innovation dans des environnements complexes et turbulents ${ }^{10}$. ». On peut alors avancer que le fonctionnement par projet peut contribuer à la mise en œuvre de la stratégie. Aujourd'hui, avec les mutations de l'environnement, la stratégie peut être mise en œuvre grâce à un portefeuille de projets sachant introduire et réaliser le changement espéré tant dans l'environnement externe que dans l'environnement interne. D'une part, le management par projet permet de s'adapter dans l'environ- nement externe en s'approchant plus du client et en essayant de le satisfaire au maximum. D'autre part, le management par projet modifie l'environnement interne de l'entreprise en transformant les pratiques traditionnelles et les comportements vers des méthodes de travail plus transversales, des structures plus souples et surtout une culture «projet» assurant une communication inter-projets et inter-fonctions afin de relever le défi de la performance à travers le triplet coût, qualité et délai.

Le management par projet modifie l'environnement interne de l'entreprise en transformant les pratiques traditionnelles et les comportements vers des méthodes de travail plus transversales, des structures plus souples et surtout une culture « projet » assurant une communication inter-projets et inter-fonctions afin de relever le défi de la performance à travers le triplet coût, qualité et délai.

\section{DU MANAGEMENT PAR PROJET AU MANAGEMENT STRATÉGIQUE PAR PROJET}

\subsection{L'approche stratégique du projet}

Appréhender les projets dans une perspective stratégique peut contribuer à mieux gérer ou digérer l'incertitude en cherchant à explorer le futur ou le faire advenir plutôt que de le deviner et le prévoir.

Le projet est doté d'une caractéristique antagoniste puisqu'il est considéré en même temps comme source d'incertitude, mais aussi comme outil d'anticipation. En effet, appréhender les projets dans une perspective stratégique peut contribuer à mieux gérer ou digérer l'incertitude en cherchant à explorer le futur ou le faire advenir plutôt que de le deviner et le prévoir. En ce sens, les projets traduisent le volontarisme managérial et le souci permanent de création d'un déséquilibre concurrentiel, éphémère par définition mais source potentielle d'un avantage stratégique. En outre, multiplier le nombre de projets au sein des organisations assouplit l'impossible exigence de justesse de la prévision $^{11}$. Dans cette perspective, le projet apparaît comme un dispositif permettant de maintenir un certain degré de réversibilité des décisions et comme un moyen de sonder et tester les marchés.

Enfin, l'approche stratégique du projet consacre l'inextricable entre l'émergent et le délibéré. Le projet constitue le lieu privilégié où se construit cette rencontre entre délibération et émergence et façonnent un cadre stratégique qui n'apparaît plus comme imposé et subi, mais au contraire comme support à la conduite d'actions de changement issues des acteurs eux-mêmes.

2.2 La mise en place de la stratégie de l'entreprise à travers les projets

L'accent mis sur la perspective stratégique des projets s'est développé davantage durant la dernière décennie que ce soit au niveau d'un projet ou d'une multiplicité de projets ou au niveau de toute l'organisation. 
L'essence du management par projet est de supporter l'exécution de la stratégie compétitive de l'organisation pour délivrer un output désiré (réponse rapide au marché, qualité supérieure, produits à bas coût). Toutefois, l'échec dans la mise en œuvre d'une stratégie est souvent dû à la direction générale qui essaie d'utiliser les systèmes et les structures organisationnelles en place (son «statu quo ») pour changer le «statu $q u o »$. Le management par projet peut remédier à certains problèmes puisqu'il réunit les meilleures compétences dans l'entreprise pour transformer les habitudes de travail et introduire un changement dans l'organisation. Aussi, parler du management par projet et son rôle dans le processus de choix et de mise en place de la stratégie revient-il à le placer par rapport aux différentes formes de la stratégie, en l'occurrence la stratégie délibérée et la stratégie émergente. Formant la stratégie explicite choisie après un effort d'analyse stratégique et d'étude des priorités, la stratégie délibérée peut être ensuite mise en œuvre à travers des équipes projets. Ainsi, la stratégie peut constituer le cadre de référence et la direction auquel doit se conformer les projets dans l'entreprise. Au fur et à mesure de sa mise en œuvre, les équipes projets évaluent davantage l'intérêt de leurs choix stratégiques, pouvant donner lieu à des stratégies émergentes qui influencent de nouveau les projets en cours et à venir.

De plus, avec l'initiative d'innovation et de créativité encouragée par le management par projet, la stratégie d'entreprise constitue un support aux projets. En effet, elle assure la cohérence et la compatibilité entre eux pour la mise en place de la stratégie et contribue à la réalisation de changements organisationnel et culturel dans l'entreprise. Comme le choix d'une stratégie et sa mise en place est une étape fondamentale s'inscrivant dans le quotidien de l'entreprise, le management par projet, étant largement lié aux choix stratégiques de la direction générale, contribue à favoriser la réactivité des entreprises face à la turbulence de l'environnement en mettant en place la stratégie délibérée et en l'évaluant grâce à un processus d'apprentissage continue. La réalisation de la stratégie de l'entreprise grâce à la mise en œuvre de projets est appropriée dans un contexte d'incertitude et de turbulence et de l'environnement. Un tel mode de gestion est qualifié de management stratégique par projet, tel que schématisé à la figure 2.

\section{Figure 2 : L'approche du management stratégique par projet}

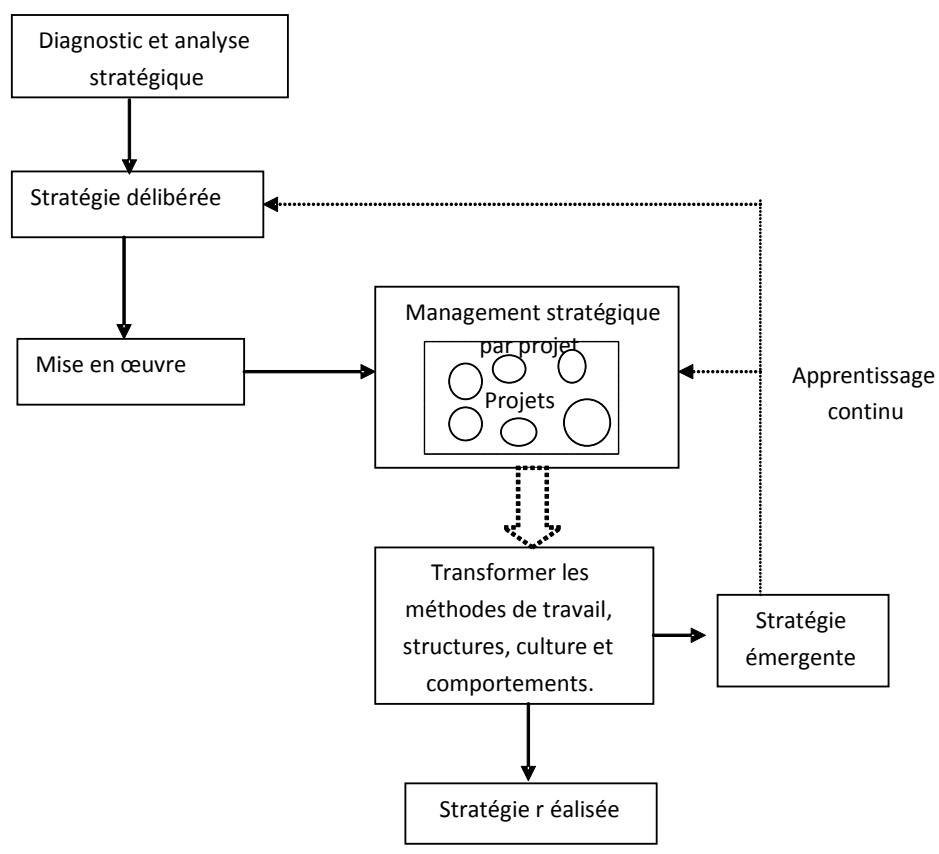


Le management par projet peut aussi aider les dirigeants à construire en interne leur plateforme stratégique inscrite dans le cadre d'une gestion stratégique des ressources et des compétences. Il s'agit de développer à la fois des compétences «métier» et de se doter des capacités organisationnelles nouvelles, lesquelles requièrent une participation active et une coopération transversale entre les acteurs au sein de l'entreprise.

Finalement, s'inscrivant dans le cadre d'une stratégie, le projet a pour objet de réussir l'implantation d'une innovation au sein d'un ensemble organisationnel permettant de répondre aux exigences du client en respectant des coûts et des échéances prédéterminés et en mettant l'emphase sur la communication entre les parties prenantes du projet. C'est la gestion de projets orientée vers le client (CustomerDriven Project Management). Cette approche flexible permet l'accomplissement d'un projet et une performance qui satisfait toutes les parties prenantes, dont les plus importantes sont les clients. À travers cette approche, la gestion par les projets s'insère davantage au service de la stratégie.

\subsection{Méthodologie du management stratégique par projet}

Par analogie à la démarche classique du management stratégique, nous proposons une méthodologie en cinq phases pour traduire la mise en place de la stratégie de l'entreprise à travers les projets dans une optique orientée vers la satisfaction des clients. Les cinq phases (conception, décision, action, contrôle et amélioration continue, intégration) sont illustrées à la figure 3.

\section{Phase de conception : Ce qui devrait se faire}

Cette phase s'entreprend par la définition des attentes des clients, la spécification des procédés et la sélection des opportunités d'amélioration. Ensuite, il s'agit d'identifier l'équipe qui doit mener le processus de gestion. Cette équipe peut comporter uniquement des directeurs supérieurs ou peut inclure des directeurs de projets, un directeur général, et peut-être un client. Ensuite, cette équipe clarifie ou développe les buts attendus de projets. C'est un pas crucial qui détermine si le reste du processus de la sélection $\mathrm{du}$ projet peut être prospère. Finalement, le choix des critères de mesures pour l'évaluation des projets aide à orienter les efforts et se concentrer sur moins de projets.

\section{Phase de la décision: Ce qui peut se faire}

Il s'agit d'organiser les projets par catégories pour faciliter le processus de la prise de décision. Différentes techniques peuvent être développées pour gérer avec la complexité dans les projets. Il est donc possible de positionner les projets sur un continuum selon l'ampleur de changement du produit et l'ampleur de changement du processus pour faciliter le contrôle. Ensuite, il faut évaluer les projets dans chaque catégorie en sélectionnant des indicateurs de mesure qui peuvent être économiques ou techniques, quantitatifs ou qualitatifs, subjectifs ou objectifs, dynamiques ou statiques, absolus ou relatifs. Finalement, il s'agit d'éliminer les projets dont la consommation de ressources dépasse les attentes initiales, où les coûts de matières sont plus élevés qu'attendus, ou dont la concurrence au marché a changé les règles du jeu.

\section{Phase de l'action : Faire}

L'équipe ou les directeurs supérieurs responsables doivent mettre en vigueur le plan de compétences adéquat. Pour les responsables de l'entreprise, la comparaison et la sélection des projets se fait aussi bien au niveau stratégique qu'au niveau exécutif pour décider le programme et l'allocation des ressources. On évalue le niveau stratégique de chaque projet potentiel d'après la grille forces, faiblesses, opportunités et menaces. Le Processus de la Hiérarchie Analytique est aussi un outil permettant de structurer une situation complexe, identifier les critères et d'autres facteurs intangibles ou concrets, mesurer les interactions parmi eux et synthétiser toute l'information pour obtenir des priorités. Les priorités peuvent être également sélectionnées dans une optique coût /avantage. Enfin développer et mettre en place les outils d'assistance à l'anticipation en intégrant notamment la gestion des risques et des menaces. 
Phase du contrôle des résultats et de l'amélioration continue : Faire mieux

Il s'agit de mesurer la performance en terme de qualité, coût et délais pour assister la prise de décision, aider au pilotage et assurer le suivi des enjeux tout en adoptant une démarche d'amélioration continue. Il faut aussi anticiper les risques d'échec prévisibles.

\section{Phase de l'intégration : Faire facilement}

Le choix d'une structure pertinente du projet et son intégration dans l'organisation de l'entreprise constituent une des conditions de réussite de cette implantation. Intégrer, ce n'est pas uniquement mettre en œuvre. Il s'agit d'intégrer les projets dans la chaîne de valeur, ce qui contribue à créer de la valeur globale autant sur les plans organisationnel, technologique que culturel.

Figure 3 : Mise en place du management stratégique par projet

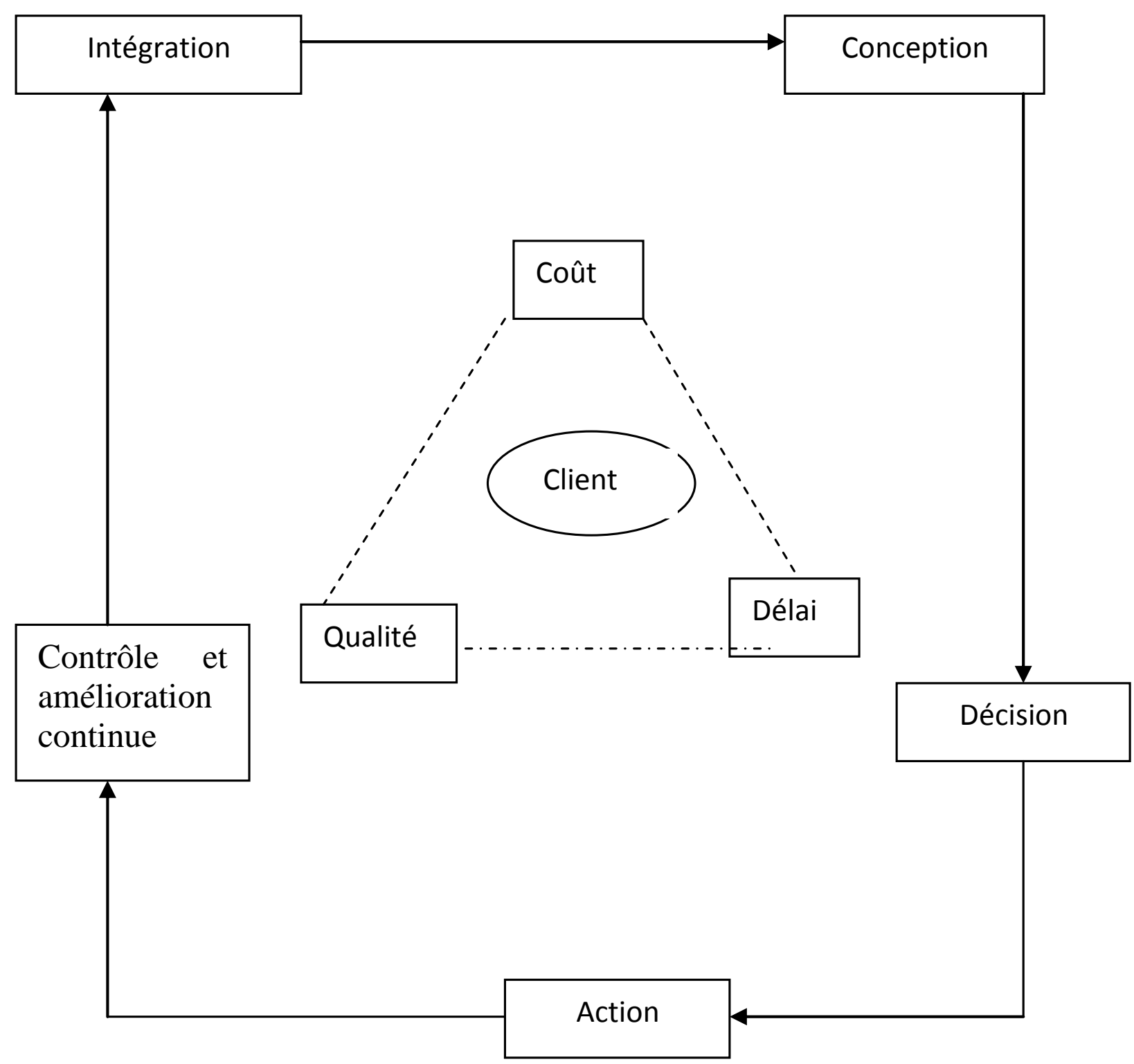




\section{CONCLUSION}

Le management par projet contribue à favoriser la réactivité des entreprises face à la turbulence de l'environnement en mettant en place la stratégie délibérée et en l'évaluant grâce à un processus d'apprentissage continu.

Cet article se donnait pour objectif de revisiter les principes d'articulation entre gestion des projets et management stratégique par projet. À cet égard, la gestion de projets est une discipline qui concerne un seul projet comportant des outils, techniques et approches organisationnelles pour gérer les relations et les comportements humains. Tandis que le management par projet concerne un ensemble de projets, voire un portefeuille de projets distincts qu'il faut intégrer avec succès au sein de l'entreprise. Le management par projet contribue à favoriser la réactivité des entreprises face à la turbulence de l'environnement en mettant en place la stratégie délibérée et en l'évaluant grâce à un processus d'apprentissage continu. Il s'agit sans doute d'une nouvelle approche organisationnelle dans la manière de concevoir le fonctionnement des entreprises en mode projet. L'alignement de projets à la stratégie permet d'atteindre les objectifs de flexibilité et une meilleure maitrise des ressources et des compétences. De plus, l'intégration des projets dans le mode d'organisation des entreprises est une compétence stratégique. Sur ce point, il est approprié de se conformer au modèle en cinq phases pour traduire la mise en place de la stratégie de l'entreprise à travers les projets dans une optique orientée vers la satisfaction des clients.

\section{L'alignement de projets à la stratégie permet d'atteindre les objectifs de flexibilité et une meilleure maîtrise des ressources et des compétences.}

\section{BIBLIOGRAPHIE}

${ }^{1}$ Shehar A.J., Dvir D. (2007). "Project Management Research - the Challenge and Opportunity". Project Management Journal, v.2, June, 93-99. Srivannaboon S., Milosevic D. Z. (2006). “A Two-Way Influence Between
Business Strategy and Project Management". International Journal of Project Management, 24, 493505.

${ }^{2}$ Zannad H. (1998). « La gestion par projet à l'épreuve des faits ». L'Expansion Management Review, décembre p. 85.

${ }^{3}$ Lan-ying D. et Yong-dong S. (2007). "Implement Business Strategy via Project Portfolio Management : A Model and Case Study". The Journal of American academy of business, vol. 11, $\mathrm{n}^{\circ}$ 2, 239-244.

${ }^{4}$ Bellanger L. et Couchaère M. J. (1992). Animer et gérer un projet. Éditions ESF, Paris, p. 65.

${ }^{5}$ Naaranoja M., Haapalainen P., Lonka H. (2007). "Strategic Management Tools in Projects Case Construction Project". International Journal of Project Management, 25, 659-665.

${ }^{6}$ Leroy D. (1996). «Le management par projet: entre mythes et réalités ». Revue Française de Gestion, janvier février, p. 110.

${ }^{7}$ Fernez-Walch S., Triomphe C. (2007). «Le management multi-projets, définitions et enjeux ». Faire de la recherche en management de projet (Garel G; Giard V., Midler C.), éditions Vuibert, 189-207.

${ }^{8}$ Declerck R. P. ; Eymery P. et Crener M. A. (1980). Le management stratégique des projets. Éditions Hommes et Techniques, Paris. Declerck R., Debourse J.-P. et Navarre C. (1983). Méthode de Direction générale: le management stratégique, Hommes et Techniques.

${ }^{9}$ Raviart, D.; Senechal, O. et Tahon, C. (1999). «Méthodologie d'évaluation de projets dès la conception ». Travail et Méthodes, $\mathrm{n}^{\circ} 544$.

${ }^{10}$ Tarondeau J. C. et Wright R. W. (1995). «La transversalité dans les organisations ou le contrôle par les processus ». Revue française de gestion, $\mathrm{n}^{\circ} 104$.

${ }^{11}$ Aurégan, P. Joffre, P. (2002). «Le projet au cœur de la stratégie » L'Expansion Management Review, 88-97. Grundy, T. (1998). "Strategy implementation and project management". International Journal of Project Management 16, 43-50. Milosevic D., Srivannaboon S. (2006). «A Theoretical Framework for Aligning Project Management with Business Strategy". Project Management Journal, Vol. 37, $\mathrm{N}^{\circ}$ 3. Morris P. W. G., Jamieson A. (2005). "Moving from corporate strategy to project strategy", Project Management Journal, 36, 4, 518. Muller R., Turner R. (2007). "The Influence of Project Managers on Project Success Criteria and Project Success by Type of Project". European Management Journal, Vol. 25, nº 4, 298-309.

Notes

${ }^{a}$ Maître assistante, Faculté des sciences économiques et de gestion. Université de Tunis, Tunisie.

'Maître assistant à l'Institut Supérieur de gestion de Tunis, Tunisie. 
2011

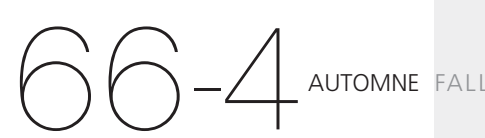

REVUE TRIMESTRIELLE

RELATIONS INDUSTRIELLES

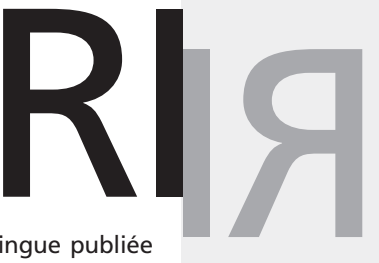

Revue trimestrielle bilingue publiée depuis 1945 par le Département des relations industrielles de l'Université Laval

NUMÉRO THÉMATIQUE / SPECIAL ISSUE

Les systèmes de représentation au travail : à la mesure des réalités contemporaines ?

CHRISTIAN BRUNELLE, ANNETTE HAYDEN ET GREGOR MURRAY

Employee Representation in the New World of Work: The Dynamics of Rights,

Voice, Performance and Power

CHRISTIAN BRUNELLE, ANNETTE HAYDEN AND GREGOR MURRAY

ARTICLES

Labour, Liberalism, and the Democratic Party: A Vexed Alliance

NELSON LICHTENSTEIN

L'évolution de la négociation collective et de ses acteurs dans six pays européens

CHRISTIAN DUFOUR ET ADELHEID HEGE

Rethinking Unionism in a Changing World of Work, Family and Community Life

BARBARA POCOCK

Organizing Women in the Spaces between Home, Work and Community

CHARLOTTE A.B.YATES

New Forms to Settle Old Scores: Updating the Worker Centre Story in the United States

JANICE R. FINE

La représentation collective des travailleurs précaires : évolution et défis contemporains

URWANA COIQUAUD

Représentation collective et citoyenneté au travail en contexte de projet MARIE-JOSÉE LEGAULT ET MARTINE D'AMOURS

\section{NDUSTRIAL RELATIONS}

\section{QUARTERLY REVIEW}

A bilingual quarterly published since 1945 by the Industrial Relations Department, Université Laval

\section{RI/IR EN LIGNE}

$\mathrm{RI} / \mathrm{IR}$ est disponible en ligne sur le site Erudit : www.erudit.org/revue/ri

Pour abonnement à la version numérique, contacter Érudit.

Pour consulter les sommaires des numéros publiés, les résumés d'articles ou pour vous abonner à la version papier visitez notre site Internet :

www.riir.ulaval.ca

\section{RI/IR ONLINE}

$\mathrm{RI} / \mathrm{R}$ is available on line on Erudit website at: www.erudit.org/revue/ri To subscribe to the online version, please contact Erudit.

Visit our website for contents listings, abstracts, or to subscribe to the print edition:

www.riir.ulaval.ca

\section{RELATIONS INDUSTRIELLES} INDUSTRIAL RELATIONS

Pavillon J.-A.-DeSève

1025, avenue des Sciences-Humaines

Bureau 3129, Université Laval

Québec (Québec) Canada

G1V 0 A6

TÉLÉPHONE : (418) 656-2468

TÉLÉCOPIEUR / FAX : (418) 656-7688

COURRIEL / E-MAIL : relat.ind@rlt.ulaval.ca

www.riir.ulaval.ca 